\title{
Espacio fiscal para salud en las Américas: ¿es suficiente el crecimiento económico?
}

\author{
Camilo Cid Pedraza, ${ }^{1}$ Mauricio Matus-López ${ }^{2}$ y Ernesto Báscolo ${ }^{1}$
}

Forma de citar

Cid Pedraza C, Matus-López M, Báscolo E. Espacio fiscal para salud en las Américas: ¿es suficiente el crecimiento económico? Rev Panam de Salud Publica. 2018;42:e86. https://doi.org/10.26633/ RPSP.2018.86

RESUMEN Objetivo. En 2014, los países miembros de la Organización Panamericana de la Salud firmaron la Estrategia para el acceso universal a salud y cobertura universal de salud. En ella, se comprometieron a aumentar el gasto público en salud hasta alcanzar la meta referencial de $6 \%$ del producto interno bruto (PIB). El objetivo de este trabajo es determinar, para cada uno de los países de la Región, si pueden alcanzar esta meta solo con crecimiento económico y, en el caso de ser posible, en qué plazos lo harían.

Métodos. Se utilizaron datos del Banco Mundial y de la Organización Mundial de Salud y se estimaron las elasticidades del gasto público en salud con respecto al PIB para cada país. Con base en el crecimiento económico real y el proyectado por el Fondo Monetario Internacional 2016-2021, se proyectó la serie de gasto y se determinó el año en el que alcanzarían 6\% del producto.

Resultados. Seis países ya han alcanzado la meta de 6\%. Los países de América Latina y el Caribe que la han logrado son aquellos que mantienen sistemas de salud únicos, basados en acceso y cobertura universales. Si se mantiene la priorización actual del gasto público en salud, tres países podrían alcanzar la meta en la próxima década. Otros cuatro países lo harían antes de medio siglo, diez en la segunda mitad y uno tendría que esperar hasta la próxima centuria. Por último, 13 países nunca alcanzarían la meta propuesta.

Conclusiones. Este análisis demuestra las limitaciones del crecimiento económico como fuente de espacio fiscal. Será necesario recurrir a otras fuentes como mayor recaudación tributaria, impuestos específicos en salud y mayor eficiencia en el gasto público, lo que demandará un diálogo social y político de los países en torno al compromiso con los principios de la salud universal.

Palabras clave Financiación de la atención de la salud; cobertura universal; recursos en salud; América Latina.

El objetivo de la Estrategia de Salud Universal de la Organización Panamericana de la Salud (OPS) es construir un camino hacia el acceso y cobertura

\footnotetext{
Organización Panamericana de la Salud, Washington D.C., Estados Unidos de América. Enviar correspondencia a Camilo Cid Pedraza, cidcam@paho.org
}

universal en salud (1-4). Para eso, se vinculan una serie de intervenciones simultáneas a la necesidad de financiamiento público en salud que aseguren la

\footnotetext{
2 Departamento de Economía, Métodos Cuantitativos e Historia Económica, Universidad Pablo de Olavide, España.
}

equidad en el acceso (2). En esta línea, los países miembros de OPS acordaron, en 2014, elevar el gasto público en salud (GPS) hasta $6 \%$ del producto interior bruto (PIB) (4). Este valor implica un aumento promedio del GPS de casi dos puntos y medio del producto, y reduciría de manera considerable la incidencia de la catástrofe financiera y

Este es un artículo de acceso abierto distribuido bajo los términos de la licencia Creative Commons Attribution-NonCommercial-NoDerivs 3.0 IGO, que permite su uso, distribución y reproducción en cualquier medio, siempre que el trabajo original se cite de la manera adecuada. No se permiten modificaciones a los artículos ni su uso comercial. Al reproducir un artículo no debe haber ningún indicio de que la OPS o el artículo avalan a una organización o un producto específico. El uso del logo de la OPS no está permitido. Esta leyenda debe conservarse, junto con la URL original del artículo. 
el empobrecimiento en los hogares debido al gasto de bolsillo en salud (5).

La importancia del gasto público en salud ha sido abordada desde los orígenes del estudio sobre inversión en capital social $(6,7)$ hasta el desarrollo de los determinantes sociales de salud (8). En este recorrido, los trabajos más recientes confirman la relación de largo plazo entre mejoras en salud, crecimiento económico y GPS (9-12).

En este marco más general, se pone de manifiesto la importancia del concepto de espacio fiscal para salud (EFS). Los primeros trabajos sobre espacio fiscal $(13,14)$ abordaron la necesidad de mayores recursos para salud en países de ingresos medios y bajos. Estos estudios, y los siguientes, definieron el EFS como la capacidad de un país para generar recursos adicionales al presupuesto público, sin dañar las finanzas del gobierno ni la estabilidad de la economía $(15,16)$. En concreto, se postularon una serie de posibles fuentes de EFS, que, a lo largo de los trabajos empíricos, se agruparon en cinco categorías: a) crecimiento económico, b) mayor recaudación tributaria, c) reasignación de presupuestos, d) eficiencia del gasto y e) recursos percibidos desde el exterior $(16,17)$.

De estas fuentes, la primera y más estudiada ha sido la de crecimiento económico. Esta postula que, si el PIB crece, también lo hace la recaudación del Estado y, si se mantiene la distribución del presupuesto, aumentan los recursos para salud.

Aunque los estudios posteriores han mostrado las limitaciones de esta fuente (18-21), se mantiene como una de las principales para los países de ingreso bajo y medio-bajo.

Esta importancia se confirma, de manera directa e indirecta, en una revisión sistemática realizada por la Organización Mundial de la Salud (OMS) para 44 países (22), en los que el crecimiento económico se aborda de tres maneras. En la mayoría de los trabajos, el análisis se realizó a través de una valoración de las condiciones macroeconómicas y fiscales $(18-21,23)$. Una segunda forma de abordar la relación entre EFS y crecimiento fueron las estimaciones de ingresos públicos realizadas por los propios gobiernos, aunque en este caso, solo estuvo disponible para períodos de dos o tres años. En tercer lugar, otro grupo de trabajos intentó cuantificar, en términos del PIB, cuál puede ser el aumento del
GPS en un horizonte de cinco o menos años. (24-26).

Sin embargo, ninguno de estos estudios evalúa hasta qué punto el crecimiento económico puede llevar a los países a alcanzar una meta determinada de GPS. Esta es la novedad del presente trabajo.

En el caso concreto de América Latina, los trabajos específicos para países confirman que el crecimiento económico es la fuente de EFS que tiene mayor factibilidad técnica y política (26-28). No obstante, al menos en un horizonte de cinco años, es insuficiente para alcanzar la meta acordada en OPS, por lo que se hace necesario obtener recursos de otras fuentes de espacio fiscal.

El objetivo de este trabajo es determinar, para cada uno de los países de la Región de las Américas, si pueden alcanzar la meta de GPS de 6\% del PIB solo con crecimiento económico y, en el caso de ser posible, en qué año lo harían.

\section{MATERIALES Y MÉTODOS}

Se realizó un estudio de tipo cuantitativo, exploratorio y longitudinal, con las fuentes y análisis que se presentan.

\section{Fuentes}

Se utilizaron tres fuentes abiertas. La primera fue la base de datos de gasto en salud de la OMS (29). De esta se obtuvieron las series anuales de GPS como porcentaje del PIB para el período 1995-2014. El GPS corresponde a los gastos corrientes y gastos de capital del gobierno (central y local), presupuestos, créditos externos y ayudas (incluidas donaciones de agencias internacionales y organizaciones no gubernamentales que ingresan al presupuesto público de los países) y fondos de seguros sociales (obligatorios) en salud. La segunda fuente fue la base de datos de indicadores de desarrollo del Banco Mundial (30). De esta se obtuvo la evolución del PIB real anual por país para el período 1995-2015. Por último, la tercera fuente corresponde a las proyecciones de crecimiento anual del PIB del Fondo Monetario Internacional (31) para el período 2016-2021.

\section{Estimación}

El análisis estadístico se realizó en cinco pasos. El primer paso fue obtener la serie de GPS y excluir del análisis aquellos países que, para el último año con información disponible (2014), habían superado la meta de 6\% del PIB.

En el segundo paso, se calcularon las elasticidades del GPS con respecto al PIB para el período 1995-2014. Para ello, se eliminaron los valores atípicos por país, con un valor-g de $\pm 1,5$ del rango intercuartílico.

En el tercer paso, se excluyeron 13 países cuyas elasticidades fueron menores que 1. Es decir, que el GPS no aumentaría en términos del PIB en ningún escenario.

En el cuarto paso, se proyectó el GPS de los países restantes según el crecimiento esperado para 2016-2021 y el crecimiento medio histórico a partir de entonces.

De esta forma, el GPS estimado, como porcentaje del PIB quedó expresado en la ecuación (a), como:

$$
\begin{aligned}
\operatorname{GPS}_{n}^{\prime} & =\left(G P S_{n} / P I B_{n}\right) \\
& =\operatorname{GPS}_{0}^{*}\left(1+r^{*} \varepsilon\right)^{n} / P I B_{0}^{*}(1+r)^{n}
\end{aligned}
$$

Donde

GPS' $_{n}$ : GPS como porcentaje del PIB

$\mathrm{n}$ : años a partir del año 0

r: tasa de crecimiento anual del PIB correspondiente a las proyecciones del FMI para 2016-2021 y la tasa histórica para el resto de los años

$\varepsilon$ : elasticidad del GPS con respecto al PIB.

Por último, en el quinto paso, se calcularon los $n$ años para alcanzar la meta de GPS del 6\% del PIB, como sigue,

$$
\begin{aligned}
& n=\log \left(k^{*} G P S_{0}^{\prime-1}\right) / \log (\mu) \\
& \text { Donde } \\
& \mu=\left(1+r^{*} \varepsilon\right) /(1+r) \\
& \mathrm{k}=0,06(6 \%) .
\end{aligned}
$$

\section{RESULTADOS}

Solo cinco países de la Región tienen un GPS mayor a 6\% del PIB: Canadá, Costa Rica, Cuba, Estados Unidos de América (EE.UU.) y Uruguay. Los restantes 30 países se encuentran por debajo del objetivo. De ellos, Colombia, Nicaragua y Panamá están a menos de un punto de lograrlo. Otros siete países se ubican más de dos puntos porcentuales, y nueve no han llegado ni a la mitad de la meta (cuadro 1).

Las proyecciones con base en el comportamiento histórico no son alentadoras. Pese a que la Región ha crecido en promedio al 2,9\% anual en los últimos 
CUADRO 1. Principales parámetros de estimación del gasto público en salud y año en el que cada país alcanzaría la meta de $6 \%$ del producto interior bruto

\begin{tabular}{|c|c|c|c|c|}
\hline País & GPS 2014 & Elasticidad & Brecha & Año \\
\hline \multicolumn{5}{|l|}{ Países que ya alcanzaron la meta } \\
\hline Canadá & 7,4 & NC & NC & 1995 \\
\hline Estados Unidos de América & 8,3 & NC & NC & 2001 \\
\hline Costa Rica & 6,8 & NC & NC & 2002 \\
\hline Uruguay & 6,1 & NC & NC & 2004 \\
\hline Cuba & 10,6 & NC & NC & 2005 \\
\hline \multicolumn{5}{|c|}{ Países que podrían alcanzar la meta antes de 2030} \\
\hline Colombia & 5,4 & 2,36 & 0,59 & 2024 \\
\hline Ecuador & 4,5 & 3,30 & 1,49 & 2026 \\
\hline Estado Plurinacional de Bolivia & 4,6 & 2,20 & 1,43 & 2027 \\
\hline República Dominicana & 2,9 & 2,61 & 3,07 & 2029 \\
\hline \multicolumn{5}{|c|}{ Países que podrían alcanzar la meta entre 2030 y 2100} \\
\hline Nicaragua & 5,1 & 1,64 & 0,90 & 2031 \\
\hline El Salvador & 4,5 & 2,13 & 1,53 & 2033 \\
\hline Chile & 3,9 & 1,97 & 2,15 & 2035 \\
\hline Dominica & 3,8 & 1,92 & 2,23 & 2052 \\
\hline México & 3,3 & 1,68 & 2,74 & 2056 \\
\hline Panamá & 5,9 & 1,10 & 0,12 & 2060 \\
\hline Brasil & 3,8 & 1,49 & 2,17 & 2064 \\
\hline Paraguay & 4,5 & 1,28 & 1,50 & 2068 \\
\hline Trinidad y Tabago & 3,2 & 1,32 & 2,83 & 2068 \\
\hline Guatemala & 2,3 & 1,62 & 3,67 & 2069 \\
\hline Haití & 1,6 & 2,95 & 4,44 & 2072 \\
\hline Honduras & 4,4 & 1,17 & 1,58 & 2091 \\
\hline Perú & 3,3 & 1,00 & 2,68 & Después de 2100 \\
\hline \multicolumn{5}{|c|}{ Países que nunca alcanzarían la meta solo con crecimiento económico } \\
\hline Antigua y Barbuda & 3,8 & $<1$ & 2,22 & Nunca \\
\hline Argentina & 2,7 & $<1$ & 3,35 & Nunca \\
\hline Bahamas & 3,6 & $<1$ & 2,40 & Nunca \\
\hline Barbados & 4,7 & $<1$ & 1,26 & Nunca \\
\hline Belice & 3,9 & $<1$ & 2,12 & Nunca \\
\hline Grenada & 2,8 & $<1$ & 3,17 & Nunca \\
\hline Guyana & 3,1 & $<1$ & 2,88 & Nunca \\
\hline Jamaica & 2,8 & $<1$ & 3,19 & Nunca \\
\hline República Bolivariana de Venezuela & 1,5 & $<1$ & 4,46 & Nunca \\
\hline San Vicente y Granadinas & 4,4 & $<1$ & 1,61 & Nunca \\
\hline Santa Lucía & 3,6 & $<1$ & 2,40 & Nunca \\
\hline St. Kitts y Nevis & 2,1 & $<1$ & 3,86 & Nunca \\
\hline Suriname & 2,9 & $<1$ & 3,06 & Nunca \\
\hline
\end{tabular}

NC, no corresponde.

Fuente: elaboración propia.

25 años, el GPS apenas si ha aumentado con respecto al PIB.

El resultado de este comportamiento es que algo menos de la mitad de los países, 13 de ellos, registra elasticidades menores que 1.

En la próxima década, solo Bolivia, Colombia y Ecuador podrían alcanzar la meta de un GPS de 6\% del PIB, mientras que Chile, El Salvador, Nicaragua y República Dominicana lo harían en la década siguiente. Perú tendría que esperar hasta el próximo siglo y el resto no del crecimiento económico, y otros diez países tendrán que esperar hasta después de 2050.

La brecha entre el GPS actual y el objetivo de $6 \%$ es importante, pero no determinante. Países con brechas elevadas como Dominica o Guatemala podrían acercarse a la meta, si mantienen una elasticidad ingreso como la registrada. Un caso especial es Haití, que cumple este mismo patrón, pero que está afectado por condiciones excepcionales de crecimiento del GPS de los últimos años. Por el contrario, países que están cerca de la meta, como Belice, no aumentarán su gasto hasta el nivel acordado solo con crecimiento económico (figura 1).

Estos resultados coinciden con los análisis de EFS de los estudios de casos internacionales (17-18, 22-24). El crecimiento económico es determinante para aumentar el gasto en salud, pero para ello es necesario que el GPS sea elástico con respecto al PIB y que se mantenga una senda de crecimiento económico. En los casos en que el GPS es inelástico, las posibilidades de crear EFS son limitadas.

\section{Análisis de los resultados por grupos de países}

Siguiendo a OPS/OMS 2017 (32), se puede establecer que el grupo de países que ha alcanzado la meta se caracteriza por dos cuestiones fundamentales: se trata de países desarrollados, como Canadá y EE.UU., que forman parte del grupo de países que han superado la meta hace mucho tiempo, o bien se trata de países que mantuvieron o transformaron sus sistemas de salud hacia sistemas únicos, integrados y de cobertura y acceso universales. Este es el caso de Costa Rica y de Cuba, ambos con sistemas universales desde mediados del siglo pasado. Cuba, como se sabe, desarrolló su sistema nacional de salud desde inicio de los 60 como una de sus políticas sociales principales. Por su parte, Costa Rica mantiene, desde 1941, la Caja Costarricense de Seguridad Social como entidad de mancomunación solidaria de recursos, que en los sesenta decidió la universalización del seguro para toda la población y en los noventa lo consolidó como un fondo único para un sistema universal de salud. Por último, el caso más reciente es el de Uruguay, que alcanzó el indicador a la par de un avance muy importante en la universalización 
FIGURA 1. Países según la brecha del GPS actual y objetivo, y año en que alcanzaría la meta con crecimiento económico

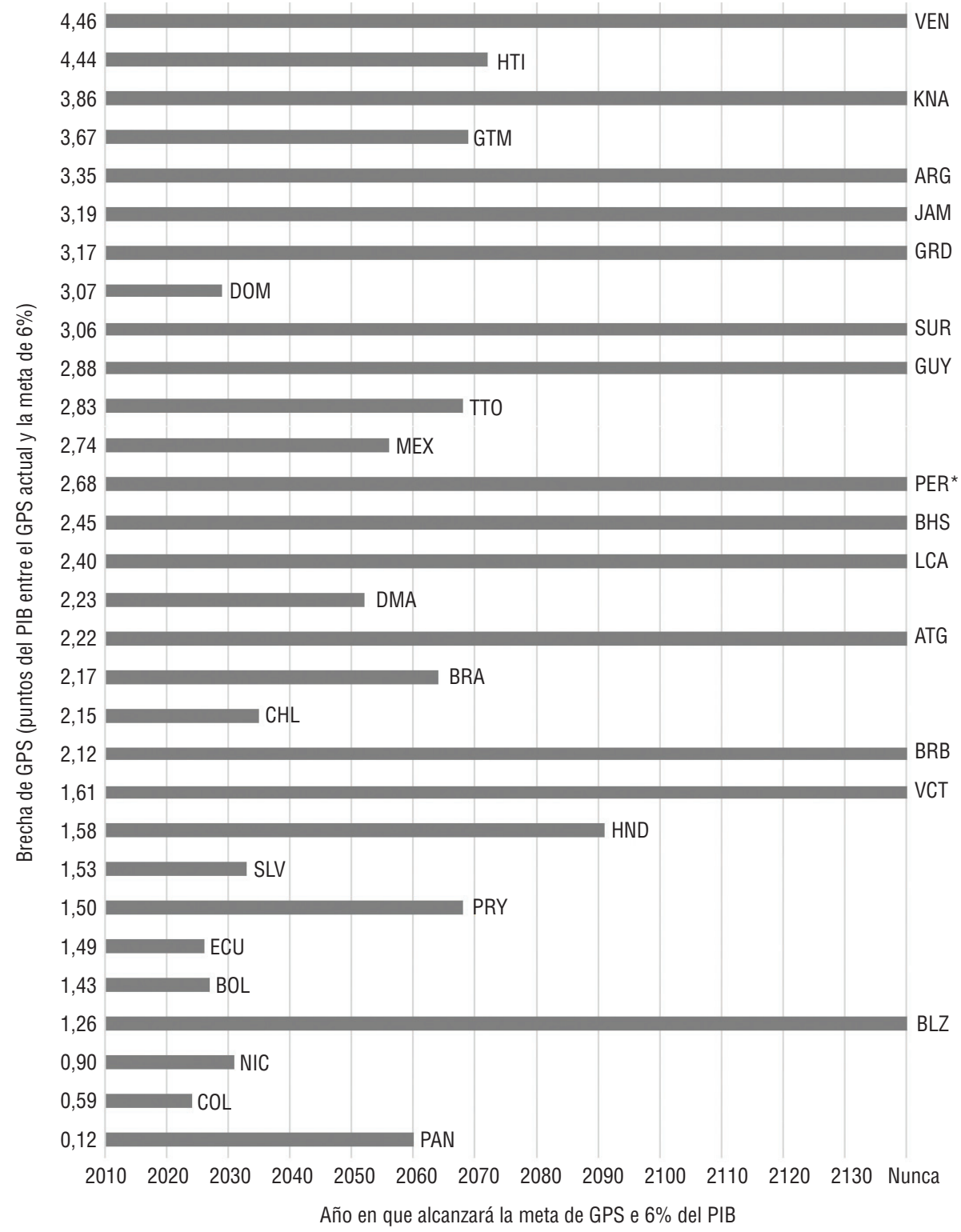

${ }^{*}$ Con posterioridad al año 2100 .

Fuente: elaboración propia.

de su sistema de salud con la reforma que comenzó a implementarse a partir de 2008 y que se caracteriza la mitigación relevante de la segmentación. Además, estos países forman parte del grupo de países con mejores resultados sanitarios de la Región.

En el segundo grupo, conformado por países que lograrían la meta antes del 2030, Bolivia y Ecuador han estado desarrollando una importante inversión pública durante los últimos años producto de políticas intencionadas que apuntan al acceso universal y equitativo por la vía del desarrollo del modelo de atención y
Dentro del grupo de 13 países que alcanzaría la meta el algún momento, un subgrupo de tres de ellos lo lograría antes de 2035 y, en realidad, se pueden caracterizar como parte del grupo anterior. En efecto, El Salvador y Nicaragua presentan políticas explícitas de aumento del acceso en los últimos años, incluida la eliminación de los copagos en el caso del El Salvador y de fortalecimiento de la gobernanza. Chile, con un gasto per cápita en salud que cuadruplica el de estos dos países, y a pesar de las reformas, es un caso más matizado en cuanto a inversión pública en salud con períodos de baja o estabilización del indicador y de alza, sin que llegue a superar 4\% del PIB.

El otro subgrupo de logro mucho más lejano (hasta el año 2100), se caracteriza por no seguir un patrón común. Se puede destacar a países con intentos de fortalecimiento del sistema y con avances en base a políticas determinadas, como Perú que ha mejorado la cobertura y el acceso, Brasil que mantiene un importante sistema del tipo servicio nacional de salud (el SUS) con importantes niveles de acceso, o México que ha intentado mejorar la cobertura con el seguro popular y a países con problemas para consolidar sus sistemas de salud, como Guatemala.

Por último, los países que presentan elasticidad del gasto en salud respecto del crecimiento del producto menor que 1 y que nunca alcanzarán la meta solo con crecimiento económico, son en su mayoría países del Caribe no latino. Estos han mantenido, en promedio, cifras bajas y estables de GPS sobre el PIB y gasto de bolsillo elevado, algunos de ellos atravesaron y aun transitan problemas macroeconómicos como el elevado endeudamiento y fueron objeto de planes de ajuste en años recientes.

De las experiencias de la Región y de otras experiencias documentadas (33) se desprende que alcanzar los éxitos en salud que supone el logro de la meta, se requiere de decisión política del más alto nivel y consenso social en la necesidad de mejorar la salud de la población con medidas y políticas decididas. El desafío para los que lo han logrado es mantener y dar sostenibilidad a los logros alcanzados, y para los que están cerca, es mantener los niveles de crecimiento de la inversión pública en salud, aún frente a posibles problemas del ciclo económico, como los que se han vivido, o cambios en el contexto político, 
lo que suele ocurrir ante los cambios de orientación de los gobiernos.

Ante los avances desiguales de los distintos componentes del financiamiento que se dan en los procesos del fortalecimiento o transformación de los sistemas, la experiencia también muestra que apelar a los instrumentos conocidos en torno al financiamiento, tales como, la mayor recaudación de impuestos, el uso de todo el potencial de la mancomunación solidaria, el conocimiento de los costos, la planificación presupuestaria efectiva y la mejora de los incentivos en la asignación de los recursos para mejorar la eficiencia, entre otras medidas, suelen ser aspectos comunes mínimos para permitir sentar las bases de avances mayores (33).

\section{CONCLUSIONES}

Los estudios específicos para países han mostrado que el crecimiento económico es una fuente con potencial técnico y factibilidad política para aumentar el GPS en la región. No obstante, su evolución y la relación entre ambas variables, establecen limitaciones. En este estudio, los resultados son contundentes: pocos países pueden alcanzar la meta de GPS igual a $6 \%$ del PIB con base en los recursos que genere el crecimiento económico. Para casi la mitad, este por sí solo no es suficiente para aumentar el gasto en

1. World Health Organization (WHO). Sustainable health financing, universal coverage and social health insurance. 58. 33 Resolution of World Health Assembly. Geneva: WHO; 2005.

2. World Health Organization (WHO). Sustainable health financing structures and universal coverage. 64.9 Resolution of World Health Assembly. Geneva: WHO; 2011.

3. Titelman D, Cetrángolo $\mathrm{O}$, Acosta OL. Universal health coverage in Latin American countries: how to improve solidarity-based schemes. Lancet. 2015;385: 1359-63.

4. Panamerican Health Organization/World Health Organization (PAHO/WHO). Strategy for Universal Access to Health and Universal Health Coverage. Resolution 53. R14 of Directing Council of Regional Comitee of World Health Organization for the Americas. Washington D.C.: PAHO; 2014.

5. Ke X, Saksena $P$, Jowett $M$, Indikadahena Ch, Kutzin J, Evans D. Exploring the thresholds of health expenditure for protection against financial risk. World Health salud, y para otro tercio, su aporte es tan limitado, que los llevaría a esperar a la segunda mitad de este siglo para lograrlo.

Este resultado, como en otros estudios, conduce a la búsqueda de recursos en fuentes de EFS adicionales, como el tipo de recaudación impositiva (34-35). Será necesario analizar otras fuentes complementarias, que en los casos específicos de América Latina también parecen señalar a la baja recaudación tributaria actual (36) y hacia mejoras en la eficiencia en el gasto público, entre otros (26-28). Estos cambios demandarán un diálogo social y político amplio en torno al compromiso de avanzar hacia la salud universal.

Las limitaciones de este trabajo tienen que ver principalmente con aquellas cuestiones que escapan al objetivo concreto de este artículo. La primera es que, más allá del gasto público en salud, existen otros problemas relevantes y específicos de cada país para alcanzar la salud universal, como la organización de los sistemas de salud, la gobernanza o la misma gestión médica, entre otros. Los mayores recursos para el sector no eliminan, por sí solos, las ineficiencias o inequidades de los sistemas nacionales. La segunda limitación es que la meta de GPS de 6\% del PIB viene dada por el acuerdo formal de los países miembros de OPS/OMS para las Américas en 2014. Si bien tiene un respaldo técnico,

\section{REFERENCIAS}

Report, Background Paper. 2010;19. Disponible en http://www.who.int /healthsystems / topics / financing / healthreport/19THE-thresv2.pdf?ua=1

6. Schultz TW. Investment in human capital. Am Econ Rev. 1961;51:1-17.

7. Mushkin SJ. Health as an investment. J Polit Econ. 1962;70:129-57.

8. Marmot M, Wilkinson R. Social determinants of health. New York: Oxford UP; 1999.

9. Herwartz H, Theilen B. The determinants of health-care expenditure: new results from semiparametric estimation. Health Econ. 2010;19:964-78.

10. Narayan PK, Narayan S, Mishra S. Investigating the relationship between health and economic growth: empirical evidence from a panel of 5 Asian countries. J Asian Econ. 2010;21:404-11.

11. Tang CF. A Note on the health-growth nexus in Malaysia. J Health Manag. 2013; 15:345-52.

12. Kuloglu A, Topcu E. The relationship between health and growth in Eurasian Economic Union. Eurasian Journal of Economics and Finance. 2016;4:42-8. alcanzar la meta no es condición que asegure los recursos suficientes para el logro de salud universal en cada uno de los países.

Con todo, el análisis por grupo permite constatar que los países que mantienen sistemas de salud con cobertura y acceso universales son los que han alcanzado la meta y que los que se acercan a ella han mantenido políticas de reforma o fortalecimiento del sector salud durante varios años.

Por último, las fuentes utilizadas tienen dos tipos de limitaciones. Por una parte, la actualización de las series de GPS es lenta y puede llegar a varios años de rezago, y puede variar, por ajustes metodológicos, según incluyan o no las cotizaciones obligatorias. En algunos pocos casos los datos sorprenden por sus cambios en el tiempo, como es el caso de Argentina y es probable que existan este tipo de dificultades.

Por otra parte, las proyecciones quinquenales de crecimiento económico pueden variar con las actualizaciones.

Conflicto de intereses. Ninguno declarado por los autores.

Declaración. Las opiniones expresadas en este manuscrito son responsabilidad del autor y no reflejan necesariamente los criterios ni la política de la RPSP/ PAJPH y/o de la OPS.
13. Heller, P. Understanding Fiscal Space. IMF Policy Discussion Paper, Issue PDP/05/4, 2005.

14. International Monetary Fund, World Bank (IMF/WB). Fiscal policy for growth and development: an interim report. DC20060003. 73 Meeting of the Development Committee. Washington DC.: IMF/WB; 2006. Disponible en http://siteresources. worldbank.org / DEVCOMMINT / Documentation / 20890698 / DC20060003(E)-FiscalPolicy.pdf Acceso el 10 de febrero de 2017.

15. Heller P. Back to basics. Fiscal space: what it is and how to get it. Finance and Development. 2005;42(2).

16. Heller PS. The prospects of creating 'fiscal space' for the health sector. Health Policy Plan. 2006;21:75-9.

17. Tandon A, Cashin C. Assessing public expenditure on health from a fiscal space perspective. Washington D.C.: World Bank; 2010.

18. William G, Hay R. Fiscal space and sustainability from the perspective of the health sector. En: WHO/WB. High Level Forum on the Health Millennium Development 
Goals: Selected papers. Geneva: Switzerland; 2006:44-66.

19. Durán-Valverde F, Pacheco JF. Fiscal space and the extension of social protection. lessons learnt from developing countries. ESS Paper Series SECSOC. 2012;33.

20. Tangcharoensathien V, Patcharanarumol W, Ir P, Aljunid SM, Mukti AG, et al. Health-financing reforms in Southeast Asia: challenges in achieving universal coverage. Lancet. 2011:377:863-73.

21. Ostry JD, Ghosh AR, Kim JJ, Qureshi MS. Fiscal space. IMF Staff Position Note. 2010. SPN/10/11. Disponible en https://www. imf.org/external/pubs/cat/longres. aspx?sk=23726.0 Acceso el 2 de febrero de 2017.

22. Marcel M. Budgeting for fiscal space and government performance beyond the great recession. OECD Journal on Budgeting. 2014;13(2):9-47.

23. Barroy H, Sparkes S, Dale E. Projecting fiscal space for health in low and middle-income countries: a review of the evidence. Geneva: WHO; 2016. Disponible en http://www. who.int/health_financing/documents/ assessing-fiscal-space/en Acceso el 1 de febrero de 2017.

24. Gupta I, Mondal S. Fiscal space for health spending in Southeast Asia. J Health Care Finance. 2013;39(4):68-82.
25. Sharma J. An assessment of fiscal space for health in Bhutan: Fiscal space for health in Bhutan. Int J Health Plann Manage. 2015;31(3):296-308. doi: 10.1002/ hpm.2295

26. Matus-Lopez M, Cid C. Una evaluación del espacio fiscal para salud en Perú. Rev Panam Salud Publica. 2016;40(1):64-9.

27. Matus-López M, Cansino D, Cid C, Valdés W. Evaluación del espacio fiscal para salud en Bolivia. Rev Panam Salud Publica. En prensa 2018.

28. Matus-López M, Cid C. Espacio fiscal para salud en América Latina y el Caribe. Washington D.C.: Organización Panamericana de la Salud; En prensa 2018.

29. World Health Organization (WHO). Global Health Observatory Data. Health Financing. Disponible en http://www.who.int/gho/ health_financing/en Acceso el 30 de marzo de 2017.

30. World Bank (WB). World Development Indicators. Disponible en http://data. worldbank.org/data-catalog/worlddevelopment-indicators Acceso el 25 de marzo de 2017.

31. International Monetary Fund (IMF) World Economic Outlook. Disponible en http://www. imf. org/external/pubs/ft/ weo/2015/01/weodata/index.aspx Acceso el 15 de marzo de 2017.
32. Organización Panamericana de la Salud/Organización Mundial de la Salud (OPS/OMS). Salud en las Américas 2017. Financiamiento de la Salud en las Américas. Washington D.C.: OPS/OMS; 2017.

33. Kutzin J,Cashin Ch,Jakab,M. Implementing Health Financing Reform. Lessons from countries in transition. Copenhagen: World Health Organization; 2010.

34. Reeves A, Gourtsoyannis Y, Basu S, McCoy D, McKee M, Stuckler D. Financing universal health coverage effects of alternative tax structures on public health systems: Cross-national modelling in 89 low-income and middle-income countries. Lancet. 2015;386(9990):274-80.

35. Yates R. Universal health coverage: Progressive taxes are key. Lancet. 2015; 386(9990):227-9.

36. Organization for Economic Cooperation and Development (OECD). Tax expenditures in OECD countries. Paris: OECD Publishing; 2010.

Manuscrito recibido el 14 de junio de 2017. Aceptado para su publicación, tras revisión, el 22 de febrero de 2018. 
ABSTRACT

Fiscal space for Health in the Americas: is economic growth sufficient?

Keywords Healthcare financing; universal coverage; health resources; Latin America.
Objective. In 2014, the Pan American Health Organization member countries signed the Strategy for Universal Access to Health and Universal Health Coverage. In it, they committed to increasing public health expenditure until reaching the benchmark of $6 \%$ of gross domestic product (GDP). The objective of this paper is to determine, for each country in the Region, if they can reach this goal by economic growth alone and, if so, how long it would take.

Methods. Using World Bank and World Health Organization data, elasticity of public health expenditure with regard to GDP was estimated for each country. Real economic growth and International Monetary Fund projections for 2016-2021 were used to project the expenditure series and determine the year each country would reach $6 \%$ of GDP.

Results. Six countries have already reached the 6\% goal. The Latin American and Caribbean countries that have achieved it are those that have single health systems, based on universal access and coverage. If current prioritization of public health expenditure is maintained, three countries could reach the goal in the next decade. Four more countries would reach it before mid-century, ten in the second half of the century, and one would have to wait until the next century. Finally, 13 countries would never reach the proposed goal.

Conclusions. This analysis demonstrates the limitations of economic growth as a source of fiscal space. Other sources will need to be tapped, such as increased tax collection, specific health taxes, and greater efficiency in public spending, which will require social and political dialogue in the countries regarding their commitment to universal health principles.
RESUMO

\section{Espaço fiscal para saúde nas Américas: 0 crescimento econômico é suficiente?}

Objetivo. Em 2014, os Estados Membros da Organização Pan-Americana da Saúde firmaram a Estratégia para o acesso universal à saúde e cobertura universal de saúde com a qual se comprometeram a aumentar o gasto público em saúde até atingir a meta de referência de $6 \%$ do produto interno bruto (PIB). O objetivo deste estudo foi determinar se cada um dos países da Região conseguiria atingir esta meta apenas com o crescimento econômico e, neste caso, em que prazo.

Métodos. O estudo se baseou em dados obtidos do Banco Mundial e da Organização Mundial de Saúde (OMS). Foi estimada a elasticidade do gasto público em saúde com relação ao PIB para cada país. A partir do crescimento econômico real e do crescimento projetado pelo Fundo Monetário Internacional para o período 2016-2021, foi feita a projeção dos gastos e determinado o ano em que seriam alcançados $6 \%$ do PIB. Resultados. Seis países já atingiram a meta de 6\%. Os países da América Latina e Caribe que atingiram esta meta são os que têm um sistema de saúde único baseado no acesso e cobertura universais. Se for mantida a priorização atual do gasto público em saúde, três países conseguiriam alcançar a meta na próxima década. Outros quatro países atingiriam a meta antes de meados do século, 10 na segunda metade deste século e um somente a alcançaria no século seguinte. E, por fim, 13 países nunca atingiriam a meta proposta.

Conclusões. Esta análise demonstra as limitações do crescimento econômico como fonte de espaço fiscal. Será necessário recorrer a outras fontes, como maior arrecadação tributária, impostos próprios para a saúde e maior eficiência no gasto público, o que demanda dos países um diálogo social e político quanto ao compromisso com os princípios de saúde universal.

Palavras-chave Financiamento da assistência à saúde; cobertura universal; recursos em saúde; América Latina. 